\title{
THE EVALUATION OF MODELLING TECHNIQUES FOR LUBRICANT CAVITAION IN THE APPLICATION OF SQUEEZE FILM DAMPERS
}

\author{
Tieshu Fan, Kamram Behdinan \\ Department of Mechanical and Industrial Engineering \\ University of Toronto \\ Toronto, Canada
}

\begin{abstract}
Squeeze film damper (SFD) is widely adopted in turbo-engines to suppress the rotor vibration. However, the prediction of SFD performance is complicated due to the inevitable occurrence of lubricant cavitation. This paper shows the application of three different cavitation algorithms for SFD with sealed conditions. In particular, the linear complementarity problem (LCP) method, which is advanced from a previous research study, is applied to compare results from the well-known methods, i.e. the $\pi$-film model and the Elrod cavitation method, for SFD executing circular centered orbits with fully degassed lubricant in the absence of oil feeding. Moreover, numerical models are developed incorporating the mentioned algorithms to predict the hydrodynamic pressure distribution over the cavitated fluid film. Results show that the conventional $\pi$-film model overestimates the cavitation region but under-estimates the reaction force.
\end{abstract} model

Keywords: SFD; Cavitation; LCP; Elrod Algorithm; $\pi$-film

\section{INTRODUCTION}

SFD is commonly integrated into aircraft turbine engines to attenuate the level of vibration. It helps to improve the rotor stability and decrease the engine vibration leading to a reduction in the cabin noise. The design parameter of an SFD is the damping capacity. There is an optimum damping value in each application, given the fact that the rotor-bearing system has multiple degrees of freedom and too much or too little damping leads to the overall damping being less effective [1]. The dynamic performance of an SFD is affected by factors such as the damper geometry, the lubricant property and the operating condition. Development of a model that accurately predicts the dynamic behavior and the damping capacity requires integrating the effects of sophisticated lubricant characteristics including the lubricant cavitation effect.

Lubricant cavitation is an ever-present phenomenon in hydrodynamic journal bearings. Typically, cavitation reduces the lubricant density, resulting in the degradation of damping capacity especially at high whirl frequencies and large journal eccentricities [2]. Cavitation in the SFD arises under three scenarios, including the entrapment of surrounding air at low hydrodynamic pressure, the release of dissolved gas in the lubricant due to the rapid change of the oscillating pressure, and the liquid vaporization at vapor pressure. Air entrapment is usually prevented by placing seals on the SFD [3]. In addition, the lubricant is regarded as well-degassed for application in a rotor-SFD system operating under steady-state conditions [4]. Consequently, only the vapor cavitation is considered for sealed SFDs under periodic motions. Vapor cavitation significantly influences the SFD load capacity, especially at high operating speeds.

For several decades, researchers have been working on the improvement of modelling techniques to accurately simulate the lubricant cavitation. The main challenge in SFDs is the identification of the cavitation boundaries, which determines the hydrodynamic pressure distribution and the hydrodynamic fluid film reaction forces. In conventional SFD models, the fluid cavitation is represented by using the $\pi$-film model (i.e. half-Sommerfeld model) [5], where the full-film extends for $\pi$ radians in the region of positive pressure. Furthermore, Gumbel suggested that the negative pressure values based on the flow equations correspond to cavitation, while the positive pressure region is invulnerable to cavitation [6]. While the Gumbel condition is readily incorporated into pressure calculations, it violates the conservation of mass in the cavitation boundary. Swift [7] and Stieber [8] suggested an enhanced boundary condition to simulate the cavitation. This condition, which is referred to as the Reynolds boundary condition, satisfies the flow continuity at the onset of the lubricant cavitation. The more sophisticated Jakobsson-Floberg-Olsson (JFO) boundary conditions $[9,10]$ provide an accurate representation of the cavitation boundaries, while maintaining the conservation of mass; however, this condition has limited application due to its computational inefficiency. The computational deficiency corresponding to the cavitation models is addressed by the Elrod algorithm [11, 12]. This model transforms the governing lubricant cavitation equation from elliptic form into parabolic form for both full film and cavitation regions. It firstly calculates the lubricant mixture density in the cavitation zone and subsequently, uses the density values to determine the hydrodynamic lubricant pressure distribution. The Elrod algorithm provides accelerated computational efficiency; however, the calculation of the film pressure and the cavitation extent substantially depend on the liquid bulk modulus. A novel lubricant cavitation evaluation technique has been 
recently introduced based on the principle of complementarity [13]. This method is further elaborated to successfully solve the cavitation problem for SFD with open-ended conditions [14].

This paper will evaluate three cavitation algorithms, i.e. the $\pi$-film model, the Elrod cavitation algorithm and the LCP method, for sealed SFD executing circular centered orbits with fully degassed lubricant in the absence of oil feeding. Firstly, the lubricant cavitation algorithms are briefly introduced, which will then be incorporated into a mathematical model to generate simulation results. Several different scenarios are subsequently studied and the results are presented for discussion.

\section{NOMENCLATURE}

\begin{tabular}{|c|c|c|}
\hline Symbol & Quantity & Unit \\
\hline C & SFD Radial Clearance & $m$ \\
\hline$F_{r}$ & Radial Force & $N$ \\
\hline$F_{t}$ & Tangential Force & $N$ \\
\hline$g$ & Switch Function & \\
\hline$h=c(1+\varepsilon \cos \theta)$ & Fluid Film Thickness & $m$ \\
\hline$L$ & SFD Length & $m$ \\
\hline$p$ & Fluid Pressure & $\mathrm{Pa}$ \\
\hline$p_{0}$ & $\begin{array}{l}\text { Pressure at Maximum } \\
\text { Film Thickness }\end{array}$ & $\mathrm{Pa}$ \\
\hline$p_{c a v}$ & Cavitation Pressure & $\mathrm{Pa}$ \\
\hline$R$ & SFD Radius & $m$ \\
\hline $\operatorname{Re}=\rho \omega \mathrm{c}^{2} / \mu$ & Squeeze-Film Reynold Number & \\
\hline$t$ & Time & $s$ \\
\hline$\beta$ & Fluid Bulk Modulus & $\mathrm{Pa}$ \\
\hline$\varepsilon$ & Eccentricity Ratio & \\
\hline$\theta$ & Angular Coordinate & rad \\
\hline$\rho$ & Fluid Density & $\mathrm{kg} / \mathrm{m}^{3}$ \\
\hline$\rho_{c}$ & $\begin{array}{l}\text { Liquid Density at } \\
\text { Cavitation Pressure }\end{array}$ & $\mathrm{kg} / \mathrm{m}^{3}$ \\
\hline $\bar{\rho}=\rho / \rho_{c}$ & Density Ratio & \\
\hline$\mu$ & Fluid Viscosity & $\mathrm{Pa} \cdot \mathrm{s}$ \\
\hline$\omega$ & Whirling Velocity & $\mathrm{rad} / \mathrm{s}$ \\
\hline$\xi$ & Complementarity Variable & $\mathrm{kg} / \mathrm{m}^{3}$ \\
\hline$\eta$ & Complementarity Variable & $\mathrm{kg} / \mathrm{m}^{3}$ \\
\hline
\end{tabular}

\section{SimUlATION METHOD}

This section describes the governing equations for the hydrodynamic pressure and the fluid film reaction forces in SFDs. Furthermore, different lubricant cavitation models are presented.

Tightly sealed SFDs allow little lubricant flow in the axial direction, such that the pressure gradient along the bearing length becomes negligible. Conventionally, the long bearing approximation (LBA) [15] is applicable to tightly sealed SFDs. Moreover, the Reynolds equation is typically applied to describe the pressure distribution in the thin film [16]. The Reynolds equation corresponding to LBA for the SFD is

$$
\frac{1}{R^{2}} \frac{\partial}{\partial \theta}\left(\frac{\rho h^{3}}{12 \mu} \frac{\partial p}{\partial \theta}\right)=\frac{\partial(\rho h)}{\partial t}
$$

Integrating (1) twice in the axial direction and applying the Sommerfeld boundary condition [16] at the maximum lubricant thickness $(\theta=0)$ yields an analytical solution for the fluid pressure. Assuming that the journal center executes circularcentered orbits (CCOs), the pressure expression becomes

$$
p=-\frac{12 \mu R^{2} \omega}{c^{2}} \frac{\varepsilon \sin \theta(2+\varepsilon \cos \theta)}{(1+\varepsilon \cos \theta)^{2}\left(2+\varepsilon^{2}\right)}+p_{0} .
$$

The subsequent cavitation models will be developed based on the assumption that $p_{0}=0$.

\section{A. $\pi$-film Model}

The $\pi$-film model [5] applies the Gumbel condition which assumes that the negative pressure that is calculated from (2) corresponds to the cavitation pressure. Accordingly, the pressure distribution is described as follows

$$
p=\left\{\begin{array}{cc}
-\frac{12 \mu R^{2} \omega}{c^{2}} \frac{\varepsilon \sin \theta(2+\varepsilon \cos \theta)}{(1+\varepsilon \cos \theta)^{2}\left(2+\varepsilon^{2}\right)} & \pi<\theta \leq 2 \pi \\
p_{c a v} & 0<\theta \leq \pi
\end{array} .\right.
$$

Furthermore, the fluid force components are determined by integrating the fluid pressure over the journal surface as

$$
\begin{aligned}
F_{r} & =\int_{\pi}^{2 \pi} p \cos \theta R L d \theta=-\frac{12 \mu R^{3} L \omega}{c^{2}} \frac{2 \varepsilon^{2}}{\left(2+\varepsilon^{2}\right)\left(1-\varepsilon^{2}\right)} \\
F_{t} & =\int_{\pi}^{2 \pi} p \sin \theta R L d \theta \\
& =2 p_{c a v} R L-\frac{12 \mu R^{3} L \omega}{c^{2}} \frac{\pi \varepsilon}{\left(2+\varepsilon^{2}\right) \sqrt{1-\varepsilon^{2}}} .
\end{aligned}
$$

\section{B. Elrod Caviation Model}

The Elrod cavitation algorithm is widely employed to calculate the effect of cavitation. Firstly, (1) is modified as

$$
\frac{1}{R^{2}} \frac{\partial}{\partial \theta}\left(\frac{\beta h^{3}}{12 \mu} g \frac{\partial \bar{\rho}}{\partial \theta}\right)=\frac{\partial(\bar{\rho} h)}{\partial t},
$$

where $\beta$ is the fluid bulk modulus defined as

$$
\beta=\rho \frac{\partial p}{\partial \rho}
$$

$\bar{\rho}$ is a dimensionless parameter, which represents the ratio between the density of fluid and the liquid density at the cavitation pressure, i.e.

$$
\bar{\rho}=\frac{\rho}{\rho_{c}} .
$$


Note that $\bar{\rho}$ is less than 1 in the cavitation zone due to the reduced density by the generated cavitation bubbles.

$g$ is a switch function defined as

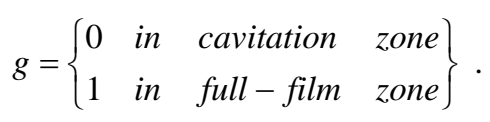

Furthermore, the switch function can be expressed by the relative density as follows to meet the required condition in both flow regions

$$
g=\frac{1}{2}+\frac{1}{2}\left(\frac{|\bar{\rho}-1|}{\bar{\rho}-1}\right) .
$$

Subsequently, numerical techniques are applied to iteratively solve $\bar{\rho}$ and $g$. The pressure distribution is calculated based on the direct integration of the bulk modulus, i.e.

$$
p=p_{c a v}+g \beta \ln \bar{\rho} .
$$

Accordingly, the reaction forces of the squeezed film are determined by integrating the hydrodynamic pressure over the journal center, i.e.

$$
\left\{\begin{array}{l}
F_{r} \\
F_{t}
\end{array}\right\}=\int_{0}^{2 \pi} p\left\{\begin{array}{l}
\cos \theta \\
\sin \theta
\end{array}\right\} L R d \theta d z
$$

\section{LCP Method}

The LCP method provides a linear complementarity equation set that can be used to efficiently find the pressure distribution.

Define two variables $\xi$ and $\eta$ as

$$
\begin{aligned}
& \xi=\rho_{c} e^{\left(p-p_{c}\right) / \beta}-\rho \\
& \eta=\rho_{c}\left[e^{\left(p-p_{c}\right) / \beta}-1\right] .
\end{aligned}
$$

It has been proved that $\xi$ and $\eta$ are non-negative and complementarity to each other in the fluid domain regardless of film rupture [14], i.e.

$$
\left\{\begin{array}{l}
\xi \geq 0 \\
\eta \geq 0 \\
\xi \eta=0 \\
\xi \frac{\partial \eta}{\partial \theta}=0
\end{array} .\right.
$$

Based on (13) \& (14), the fluid density and pressure can be described in terms of $\xi$ and $\eta$ as

$$
\begin{gathered}
\rho=\rho_{c}+\eta-\xi \\
p=p_{c}+\beta \ln \left(1+\frac{\eta}{\rho_{c}}\right) .
\end{gathered}
$$

Accordingly, the pressure gradient can also be derived from (17) as

$$
\frac{\partial p}{\partial \theta}=\frac{\partial p}{\partial \eta} \frac{\partial \eta}{\partial \theta}=\frac{\beta}{\rho_{c}+\eta} \frac{\partial \eta}{\partial \theta} .
$$

Substitution of (16)-(18) to (1) yields the following:

$$
\frac{\partial}{R^{2} \partial \theta}\left[\frac{\beta h^{3}\left(\rho_{c}+\eta-\xi\right)}{12 \mu\left(\rho_{c}+\eta\right)} \frac{\partial \eta}{\partial \theta}\right]=\frac{\partial\left(\rho_{c}+\eta-\xi\right) h}{\partial t} .
$$

The above equation can be reduced to the following after applying the complementarity condition described in (15):

$$
\frac{\partial}{R^{2} \partial \theta}\left[\frac{\beta h^{3}}{12 \mu} \frac{\partial \eta}{\partial \theta}\right]=\frac{\partial(\eta-\xi) h}{\partial t}+\rho_{c} \frac{\partial h}{\partial t} .
$$

Consequently, an LCP equation set is formulated with respect to the introduced complementarity variables after applying the finite difference method, i.e.

$$
\left\{\begin{array}{l}
\{\eta\}=[M]\{\xi\}+\{q\} \\
\{\eta\} \geq 0 \\
\{\xi\} \geq 0 \\
\{\eta\}\{\xi\}^{T}=0
\end{array},\right.
$$

where the detailed description of (21) is provided in the Appendix.

A conventional technique for solving (21) is the Lemke's pivoting algorithm [17]. Once the pressure distribution is calculated, the fluid film reaction forces are determined based on (12).

\section{CASE StUdy AND Results}

This section represents results for the three lubricant cavitation models that were discussed in the previous section. The simulation condition is described as follows: the bearing diameter is $104.9 \mathrm{~mm}$; the damper length is $110.2 \mathrm{~mm}$; the radial clearance is $0.127 \mathrm{~mm}$; the lubricant viscosity is $0.005 \mathrm{pa} \cdot \mathrm{s}$; the fluid bulk modulus is $0.069 \mathrm{GPa}$; the cavitation pressure is $1 \mathrm{kPa}$; the journal executes $\mathrm{CCO}$ and the whirling speed is $3000 \mathrm{rpm}$. Based on the values of the system parameters, the squeeze Reynolds number is at 0.9935. Large squeeze Reynolds number (i.e. $\mathrm{Re}>1$ ) requires extra nonlinear expressions to modify the Reynolds equation to address the fluid flow, which opens the gate to study the effect of the fluid inertia. In our study scenario, this number is less than 1 , thus the effect of fluid inertia is neglected so that the Reynolds equation is applied [16]. Subsequently, the three cavitation algorithms are incorporated into a MATLAB simulation model using the finite difference method.

Fig. 1-4 compares the pressure distribution for the three cavitation models under different eccentricity ratios. In general, the $\pi$-film model predicts the largest cavitation zones and the smallest peak pressure amplitudes, while the LCP method provides the largest full-film regions and the highest peak pressures. As the eccentricity ratio increases, the extent of 
cavitation based on the $\pi$-film model remains unchanged due to the corresponding assumption for the cavitation boundary condition; however, the extent of the cavitation deteriorates for the other two models. Moreover, the discrepancy between Elrod algorithm and LCP method results becomes smaller at high eccentricity ratios (i.e. $\varepsilon=0.7$ ).

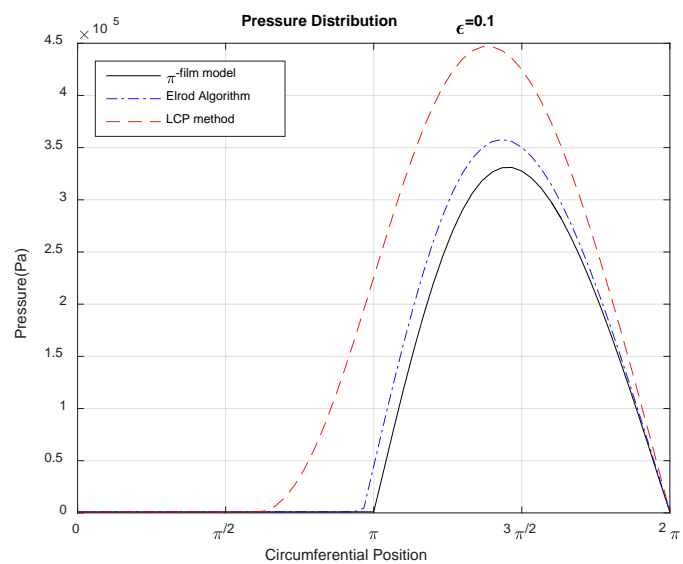

Figure 1. Comparison between the pressure distributions for the three cavitation models at eccentricity ratio 0.1

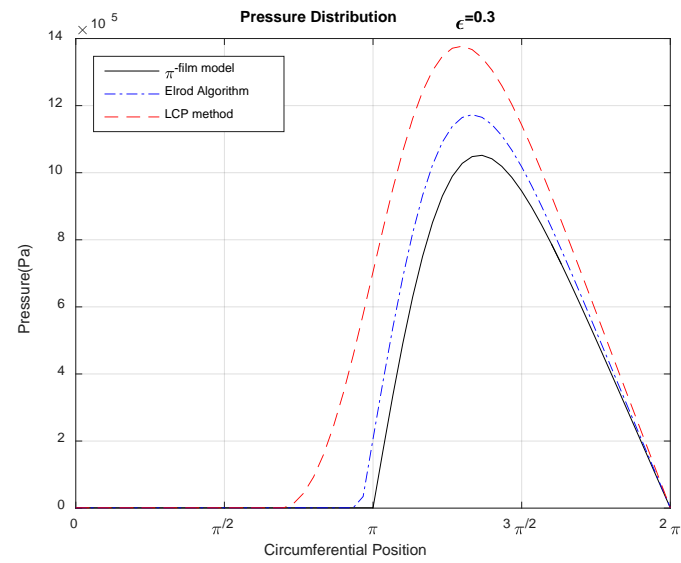

Figure 2. Comparison between the pressure distributions for the three cavitation models at eccentricity ratio 0.3

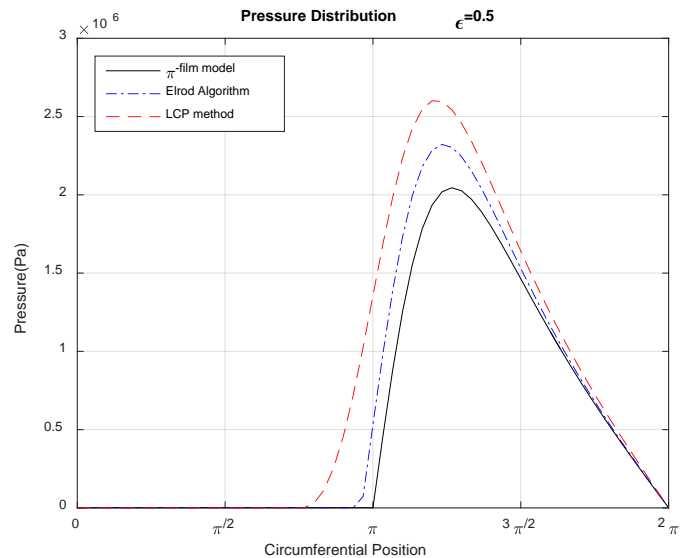

Figure 3. Comparison between the pressure distributions for the three cavitation models at eccentricity ratio 0.5

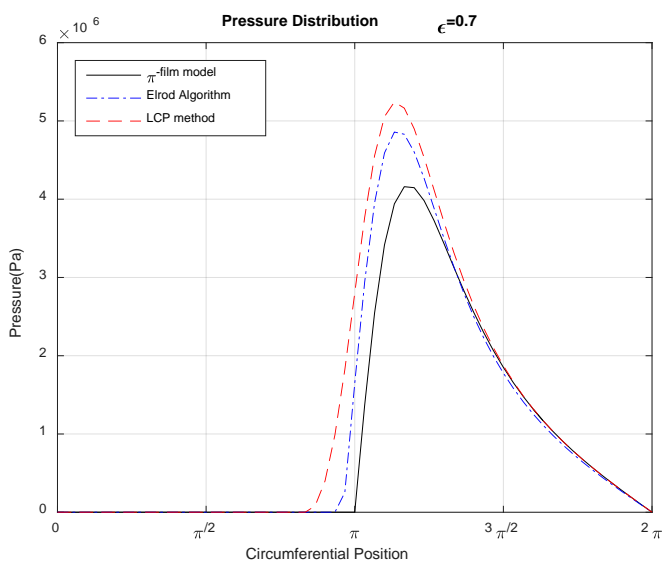

Figure 4. Comparison between the pressure distributions for the three cavitation models at eccentricity ratio 0.7

Fig. 5 and Fig. 6 compare the fluid film reaction force components at different journal eccentricity ratios. Fig. 5 shows that the magnitude of the radial force increases nonlinearly with the eccentricity ratio. Meanwhile, the $\pi$-film model predicts smaller magnitudes of the radial forces, while the LCP method presents the largest force components. Furthermore, Fig. 6 indicates a quasi-linear increase of the magnitude for the tangential force with the eccentricity ratio. The $\pi$-film model maintains the lowest predicted force magnitudes and the LCP method shows the largest force magnitudes. The discrepancies among the models are the result of differences in the pressure prediction, which can be attributed to the different assumptions about the cavitation boundary.

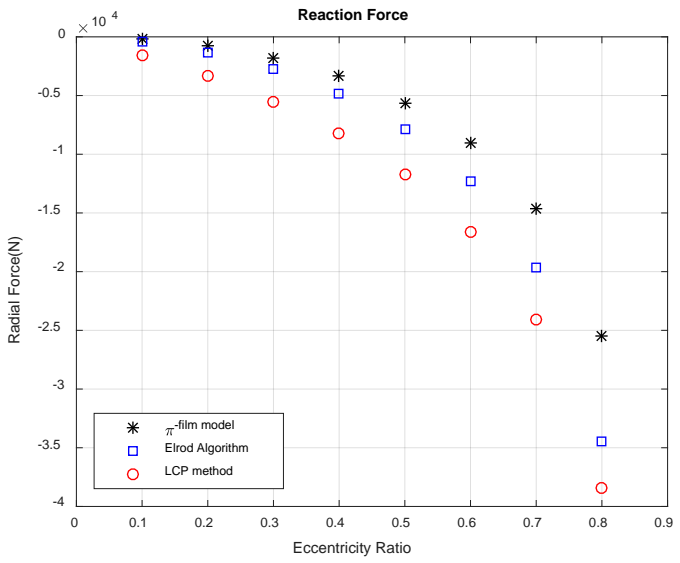

Figure 5. Comparison between the radial fluid film reaction forces for the three cavitation models at different eccentricity ratios 


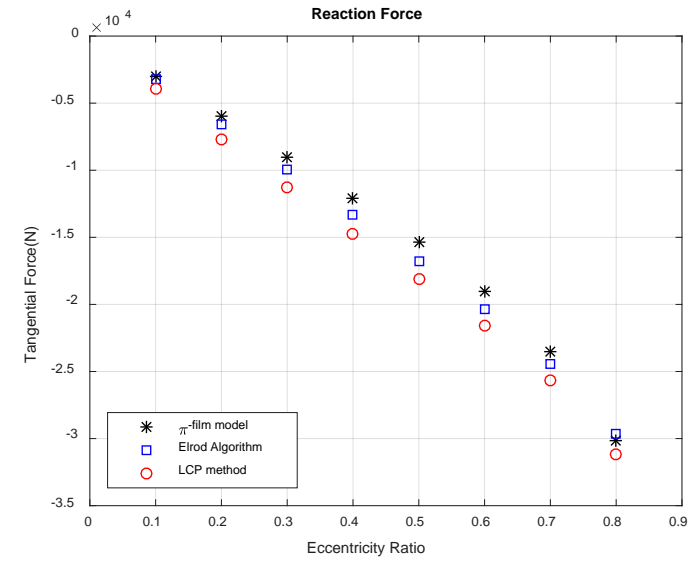

Figure 6. Comparison between the tangential fluid film reaction forces for the three cavitation models at different eccentricity ratios

Fig. 7 compares the attitude angle for the cavitation models. The attitude angel is calculated as:

$$
\theta=\arctan \left(-F_{t} / F_{r}\right) .
$$

In general, the attitude angle declines as the journal eccentricity increases. The LCP method provides the prediction of the smallest angle. Furthermore, the discrepancy between the models decreases at higher amplitude motions.

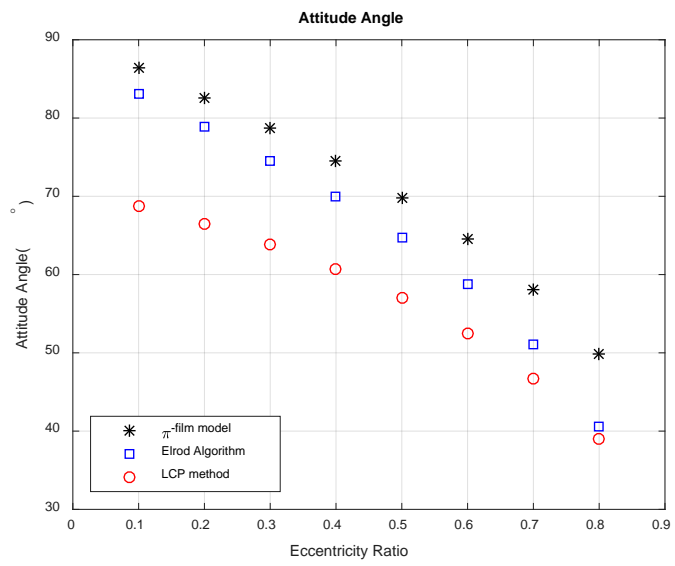

Figure 7. Comparison between the attitude angles for the three cavitation models at different eccentricity ratios

\section{CONCLUSION}

This work compares three cavitation models for sealed SFD, namely the $\pi$-film model, the Elrod algorithm, and the LCP method. Simulation shows that the extent of cavitation region is superior at large eccentricities, which is associated with the rapid buildup of larger magnitude pressure. Furthermore, the Elrod algorithm and the LCP method provide larger reaction forces but smaller attitude angles than that from the simple $\pi$ film model. These models are computationally efficient so they have the potential to be integrated into rotordynamic studies.

\section{ACKNOWLEDGMENT}

This work is supported by grants from Natural Science and Engineering Research Council (NSERC) and Pratt and Whitney Canada.

\section{REFERENCES}

[1] L. San Andres, "Squeeze film dampers: operation, models, and technical issues”, Modern Lubrication Theroy, Notes 13, Texas A\&M University Digital Libraries, College Station, TX, 2010.

[2] S. Diaz and L. San Andres, "Reduction of the dynamic load capacity in a squeeze film damper operating with a bubbly lubricant", Journal Of Engineering for Gas Turbines and Power, vol. 121, pp. 703-709, 1999.

[3] L. San Andres and A. Delgado, "Identification of force coefficients in a squeeze film damper with a mechanical end seal - centered circular orbit tests”, Journal of Tribology, vol. 129, pp. 660-668, 2007.

[4] F. Zeidan and J. Vance, "Cavitation regimes in squeeze film dampers and their effect on the pressure distribution", Tribology Transaction, vol. 33, pp. 447-453, 1990.

[5] A. Sommerfeld, A, "Zur Hydrodynamischen theorie der Schmiermittelreibung (On the hydrodynamic theory of lubrication)", Zeit. Math. Phys., vol. 50, pp. 97-155, 1904.

[6] L. Gumbel, “Monatsblatter Berliner Bezirksver”, VDI, vol. 5, pp. 87-104, 1914.

[7] H. W. Swift, "The stability of lubricating film in journal bearings", Proceedings Institute Civil Engineers, London, vol. 233, pp. 267-288, 1932.

[8] W. Stieber, “Das Schwimmlager”, VDI., Berlin, 1933.

[9] B. Jakobsso and L. Floberg, "The finite journal bearing, considering vaporization”, Chalmers Tekniska Hogskolas Handlingar, Goteborg, Sweden, vol. 189, pp. 1-116, 1957.

[10] K. O. Olsson, "Cavitation in dynamically loaded bearings", Chalmers Tekniska Hogskolas Handlingar, Goteborg, Sweden, vol. 308, pp. 1-60, 1965.

[11] H. G. Elrod and M. Adams, "A computer program for cavitation and starvation problems", Cavitation and Related Phenomena in Lubrication, ImechE, England, vol. 103, pp. 37-42, 1974.

[12] H. G. Elrod, “A cavitation algorithm”, Journal of Lubrication Technology, vol. 103, pp. 350-354, 1981.

[13] M. Giacopini, M. T. Fowell, D. Dini and A. Strozzi, "A mass conserving complementarity formulation to study lubricant films in the presence of cavitation”, Journal of Tribology, vol. 132, pp. 041702-1, 2010.

[14] T. Fan and K. Behdinan, "The evaluation of linear complementarity problem method in modeling the fluid cavitation for squeeze film damper with off-centered whirling motion”, Lubricants, vol. 5, pp. 46, 2017.

[15] M. M. Dede, M. Dogan and R. Holmes, "The damping capacity of a sealed squeeze film bearing”, Journal of Tribology, vol. 107, pp. 411418, 1985.

[16] A. Z. Szeri, "Fluid film lubricant: theory and design", Cambridge University Press, 2005.

[17] R. W. Cottle, J. S. Pang, and R. E. Stone, "The linear complementarity problem”, Report No. 60, SIAM, Philadelphia, PA, 2009. 


\section{APPENDIX}

The details of (21) are described as follows.

Since the journal executes CCOs, the time variation can be expressed as the partial variation in the circumferential direction, i.e.

$$
\frac{\partial f}{\partial t}=-\omega \frac{\partial f}{\partial \theta}
$$

where $f$ represents a function. Accordingly, (20) can be written as

$\frac{\partial}{R^{2} \partial \theta}\left[\frac{\beta h^{3}}{12 \mu} \frac{\partial \eta}{\partial \theta}\right]=-\omega \frac{\partial h}{\partial \theta}(\eta-\xi)-\omega h \frac{\partial(\eta-\xi)}{\partial \theta}-\omega \rho_{c} \frac{\partial h}{\partial \theta}$

After applying the finite difference method to the above, the LCP equation is formulated as (21), where

$$
\left.\begin{array}{c}
\{\eta\}=\left\{\begin{array}{lll}
\eta_{1} & \ldots & \eta_{N}
\end{array}\right\}^{T} \\
\{\xi\}=\left\{\begin{array}{lll}
\xi_{1} & \ldots & \xi_{N}
\end{array}\right\}^{T} \\
\{M]=[L]^{-1}[N]
\end{array}\right\}\left\{\begin{array}{c}
\rho_{c}\left(e^{\left(p_{0}-p_{c a v}\right) / \beta}-1\right) \\
-\omega \rho_{c} \frac{\partial h_{2}}{\partial \theta} \\
\vdots \\
\{s\}=\left\{\begin{array}{c}
]^{-1}\{s\} \\
-\omega \rho_{c} \frac{\partial h_{n}}{\partial \theta} \\
\rho_{c}\left(e^{\left(p_{0}-p_{c a v}\right) / \beta}-1\right)
\end{array}\right\}
\end{array}\right.
$$

$$
[L]=\left[\begin{array}{ccccc}
1 & 0 & & & \\
a_{2}-b_{2}-d_{2} & -2 a_{2}+b_{2}+c_{2}+d_{2} & a_{2} & \ddots & \\
& \ddots & \ddots & \ddots & \\
& & a_{n}-b_{n}-d_{n} & -2 a_{n}+b_{n}+c_{n}+d_{n} & a_{n} \\
& & & 0 & 1
\end{array}\right]
$$

$$
\begin{aligned}
& {[N]=\left[\begin{array}{ccccc}
1 & 0 & & & \\
-d_{2} & c_{2}+d_{2} & 0 & & \\
& \ddots & \ddots & \ddots & \\
& & -d_{n} & c_{n}+d_{n} & 0 \\
& & & 0 & 1
\end{array}\right]} \\
& a_{i}=\frac{\beta\left(h_{i}\right)^{3}}{12 \mu R^{2}(\Delta \theta)^{2}} \\
& b_{i}=\frac{\beta\left(h_{i}\right)^{2}}{4 \mu R^{2} \Delta \theta} \frac{\partial h_{i}}{\partial \theta} \\
& c_{i}=\omega \frac{\partial h_{i}}{\partial \theta} \\
& d_{i}=\frac{\omega h_{i}}{\Delta \theta} \\
& e_{i}=\omega \rho_{c} \frac{\partial h_{i}}{\partial \theta}
\end{aligned}
$$

The subscript $i(i=1,2, \ldots, \mathrm{n})$ denotes the nodal position in the discretized circumferential domain.

The first row and the last row in the LCP equation represent the boundary condition given the pressure at the maximum film thickness. 